\title{
Das Exposom charakterisiert die Auswirkungen unserer Umwelt auf Stoffwechsel und Gesundheit
}

\section{The Exposome Characterizes the Impact of Environmental Factors on Metabolism and Health}

\section{(ㄷ) (2) (-) (5)}

\author{
Autoren \\ Institute \\ 1 Zentrum für Kardiologie, Kardiologie I, Universitätsmedizin \\ der Johannes Gutenberg-Universität Mainz, Mainz, \\ Deutschland \\ 2 Deutsches Zentrum für Herz-Kreislauf-Forschung (DZHK), \\ Standort Rhein-Main, Mainz, Deutschland \\ 3 Abteilung Atmosphärenchemie, Max-Planck-Institut für \\ Chemie, Mainz, Deutschland
}

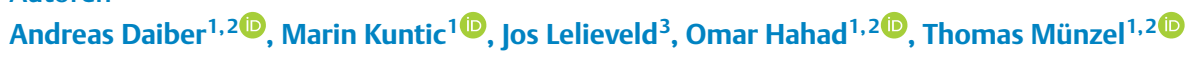

Schlüsselwörter

chronische nicht-übertragbare Erkrankungen, Umweltrisikofaktoren, Exposom, umweltbedingte kardiovaskuläre Erkrankungen

Key words

chronic non-communicable diseases, environmental risk factors, exposome, environment-related cardiovascular diseases

\section{Bibliografie}

Aktuel Kardiol 2021; 10: 502-508

DOI 10.1055/a-1546-7401

ISSN 2193-5203

(c) 2021. The Author(s).

This is an open access article published by Thieme under the terms of the Creative Commons Attribution-NonDerivative-NonCommercial-License, permitting copying and reproduction so long as the original work is given appropriate credit. Contents may not be used for commercial purposes, or adapted, remixed, transformed or built upon. (https://creativecommons.org/licenses/by-nc-nd/4.0/).

Georg Thieme Verlag KG, Rüdigerstraße 14,

70469 Stuttgart, Germany

Korrespondenzadresse

Prof. Andreas Daiber

Zentrum für Kardiologie, Kardiologie I

Universitätsmedizin der Johannes Gutenberg-Universität

Mainz

Langenbeckstraße 1

55131 Mainz, Deutschland

daiber@uni-mainz.de

\section{ZUSAMMENFASSUNG}

Aktuelle Befunde der großen globalen epidemiologischen Studien wie dem „Global Burden of Disease“-Projekt legen nahe, dass bereits jetzt nahezu $2 / 3$ der jährlichen globalen Todesfälle ursächlich auf chronische nicht übertragbare Erkrankungen wie Atherosklerose oder Diabetes zurückzuführen sind. Dies liegt vor allem an der Überalterung der Menschen in westlichen Gesellschaften, aber auch einer zunehmenden Belastung durch Boden-, Wasser- und Luftverschmutzung, Lärmbelastung, mentale Stressfaktoren und weitere Umweltrisikofaktoren. Vor etwa 15 Jahren wurde das Exposom-Konzept etabliert, um die Auswirkungen unserer Umwelt auf die Gesundheit zu erforschen. Das Exposom beschreibt dabei die Summe aller Expositionen, die lebenslang auf uns einwirken und dabei biochemische und metabolische Prozesse in unserem Körper überwiegend ungünstig verändern und so zu Gesundheitsschäden und frühzeitigen Todesfällen beitragen. Mit dieser Übersicht wird das Exposom-Konzept anhand von ausgewählten Studien erklärt und seine Bedeutung für die zukünftige Gesundheitsforschung sowie die präventive Medizin, vor allem im Hinblick auf kardiovaskuläre Erkrankungen und Therapie, erläutert.

\section{ABSTRACT}

Current results of large and global epidemiological studies such as the Global Burden of Disease project suggest that in the year 2020 almost $2 / 3$ of the annual global deaths are caused by chronic non-communicable diseases (e.g. atherosclerosis or diabetes). This is mostly due to the aging of people in western societies but is also secondary to the increasing exposure to soil, water and air pollution, traffic noise, mental stress factors and other environmental risk factors. Fifteen years ago the exposome concept was established to study the impact of our environment on health in more detail. The exposome describes the mostly harmful biochemical and metabolic changes that occur in our body by the total of different environmental exposures at a lifelong time scale and ultimately lead to adverse health effects and premature deaths. With the present overview we explain the exposome concept with the help of selected studies and highlight its relevance for future health research as well as preventive medicine, especially with respect to cardiovascular diseases and therapy. 


\section{Abkürzungen}

DALYs Disability-adjusted Life Years

EWAS Environment-Wide Association Study

OMICs Wortneuschöpfung aus dem Forschungsbereich der Biologie; dient als Oberbegriff für verschiedene molekulare Methoden bzw. biologische Disziplinen (z. B. genomics, transcriptomics, proteomics, metabolomics, secretomics etc.) die auf "-omic" enden

\section{WAS IST WICHTIG?}

Umweltrisikofaktoren sind für einen Großteil der weltweiten vorzeitigen Todesfälle und verlorenen gesunden Lebensjahre (DALYs) verantwortlich. Aus diesem Grund wurden die bislang eher wenig in klinische Richtlinien integrierten Umweltstressoren unter dem „Exposom“-Konzept zusammengefasst, um die Forschung über umweltbedingte Erkrankungen zu forcieren. Das Exposom umfasst dabei die Auswirkungen der lebenslangen Exposition gegenüber der Gesamtheit der Umweltfaktoren, wie alle Arten der Verschmutzung, Lärm, Klima, Lebensstil, aber auch das soziale Umfeld, auf unsere biologischen und biochemischen Systeme und unsere Gesundheit.

\section{Hintergrund}

Aktuelle Befunde der großen globalen epidemiologischen Studien wie dem „Global Burden of Disease“-Projekt legen nahe, dass bereits jetzt nahezu $2 / 3$ der jährlichen globalen Todesfälle ursächlich auf nicht übertragbare chronische Erkrankungen wie Atherosklerose oder Diabetes zurückzuführen sind [1]. Der Rückgang der Bedeutung der übertragbaren (infektiösen) Erkrankungen kann über eine generelle Verbesserung der Hygiene sowie der grundlegenden medizinischen Versorgung, auch in Ländern mit niedrigen Einkommen, erklärt werden. Die Zunahme des Beitrags der nicht übertragbaren chronischen Erkrankungen liegt vor allem an der Überalterung der westlichen Gesellschaften und Umweltrisikofaktoren wie der Boden-, Wasser- und Luftverschmutzung, Lärmbelastung und mentalen Stressfaktoren [2].

Während die wissenschaftlichen Anstrengungen über die letzten Jahrzehnte sich auf traditionelle kardiovaskuläre Risikofaktoren wie Diabetes, Bluthochdruck oder Übergewicht konzentriert haben, zeigen neue globale Untersuchungen, dass Umweltfaktoren wie die Luftverschmutzung maßgeblich zur Gesundheitsbelastung der Weltbevölkerung durch nicht übertragbare Erkrankungen beitragen [1, 2]. Die „Lancet Commission on Pollution and Health“ zog in ihrem Bericht die Schlussfolgerung, dass die chemische Verschmutzung der Umwelt einer der wichtigsten Risikofaktoren für chronische Erkrankungen und die Übersterblichkeit darstellt - mit ca. 9 Millionen vorzeitigen Todesfällen verursachte im Jahr 2015 die Umweltverschmutzung 16\% der weltweiten Gesamttodesfälle - 3-mal mehr als AIDS, Tuberkulose und Malaria zusammen [2]. Eine ähnliche Zahl wurde von der Weltgesundheitsorganisation (WHO) berichtet, die von einer globalen Übersterblichkeit von
12,6 Millionen im Jahr 2012 durch ein Leben unter ungesunden Umweltbedingungen ausgeht. In den am stärksten betroffenen Ländern mit niedrigen Einkommen wird 1 von 4 Todesfällen durch Umweltrisikofaktoren verursacht. Laut einer Schätzung des „Global Burden of Disease“-Projekts sind alle Formen der Umweltverschmutzung jährlich für 268 Millionen verlorene gesunde Lebensjahre (DALYs) weltweit verantwortlich [3]. Luftverschmutzung als die wichtigste Form chemischer Umweltbelastung hat Schätzungen zufolge im Jahr 2013 ca. 3,5\% der Gesamtkosten in den Gesundheitssystemen reicher Länder verursacht und sogar 7,4\% in armen Ländern [2]. Allerdings muss berücksichtigt werden, dass die gesamten sozioökonomischen Kosten durch Luftverschmutzung über die reine Belastung des Gesundheitssystems hinausgehen, da auch die verlorene Produktivität durch Krankheitstage und Frührente der exponierten Personen beachtet werden müssen. Berücksichtigt man die Co-Lokalisation von Umweltexpositionen wie Luftverschmutzung, Verkehrslärm, künstliches Licht bei Nacht, aber auch psychosoziale Faktoren wie mentaler Stress in großen Städten oder urbanen Großräumen [4], so könnte der potenzielle Nutzen einer Reduktion mehrerer Umweltrisikofaktoren die bisher berechneten sozioökonomischen Einsparungen bei Weitem übertreffen.

Die Umweltverschmutzung bildet mit anderen Umweltrisikofaktoren aus den Bereichen Lebensstil (z. B. Rauchen, Ernährung, körperliche Aktivität), klimatischen Bedingungen (z. B. hohe Temperatur und Luftfeuchtigkeit, starke UV-Strahlung) und dem sozialen Umfeld (z. B. Vereinsamung, Stress am Arbeitsplatz) die Summe aller Umweltexpositionen, die unter dem Begriff „Exposom“ zusammengefasst werden und unseren biochemischen Phänotyp und damit unsere Gesundheit stark beeinflussen. So haben alle $\mathrm{Ar}$ ten von chemischer Umweltverschmutzung in der Luft, im Boden und im Wasser, aber auch physikalische Expositionen wie Lärm, künstliches Licht im Freien bei Nacht oder klimatische Parameter und mentale Umweltstressoren nachgewiesene negative Auswirkungen auf unsere biologischen und biochemischen Systeme wie z.B. metabolische Prozesse, Enzündungskaskaden, das Redoxgleichgewicht und die zirkadiane Rhythmik [5].

\section{KURZGEFASST}

Umweltrisikofaktoren (Expositionen) tragen zu einem Großteil der weltweiten verlorenen gesunden Lebensjahre (DALYs) und Todesfälle bei.

\section{Eine neue Initiative: die „Environment-Wide Association Study“" (EWAS)}

Trotz dieser dramatischen Zahlen von internationalen Expertengremien und der wissenschaftlichen Evidenz, dass Umweltrisikofaktoren einen maßgeblichen gesundheitspolitischen Belang darstellen, wurde diesen Umweltexpositionen bislang kaum Aufmerksamkeit in den globalen Strategiepapieren für Prävention und Gesundheitspolitik geschenkt [2]. Zudem verblassen die Ausgaben, die in die Erforschung von Umweltgesundheitsrisiken investiert wurden, angesichts der astronomischen Kosten, die durch die $\mathrm{Er}$ forschung des menschlichen Genoms und der Korrelation dieser 
Daten mit vererblichen Erkrankungen (GWAS = Genome-wide Association Study) entstanden sind. Dies ist insbesondere unverständlich angesichts aktueller Schätzungen, dass die Belastung der Bevölkerung durch umweltbedingte chronische, nicht übertragbare Krankheiten deutlich höher ist als die erblich bedingte Krankheitslast [6]. Aus diesem Grund fordern nun immer mehr führende Umwelt- und Gesundheitswissenschaftler, dass in Analogie zur GWAS-Initiative ein Projekt ins Leben gerufen wird, das sich vorrangig mit umweltbedingten Erkrankungen durch verschiedenste Expositionen befasst (EWAS $=$ Environment-Wide Association Study) [7].

Eine derartige Studie, welche die Summe aller Expositionen über die gesamte Lebensspanne vieler Individuen misst und mit den biochemischen Veränderungen unserer intrinsischen Systeme sowie Gesundheitseffekten und Sterblichkeit in der Studienpopulation korreliert, wäre heutzutage noch eine große Herausforderung [7, 8]. Der technologische Fortschritt wird hochauflösende Messungen multipler Expositionen mittels Satelliten- und Bodenmessstationen-gestützter Smartphone-Apps oder durch autonome tragbare Umweltsensoren ermöglichen [9]. Unsere sozialen Aktivitäten und unser Lebensstil werden zunehmend durch die Präsenz auf sozialen Medienplattformen erfasst. Auch eine preiswerte und engmaschige Bestimmung der wichtigsten Giftstoffe und Biomoleküle in unserem Organismus mittels sogenannter OMICs-Methoden (Pollutom, Adduktom, Metabolom, Proteom, Transkriptom/ Epigenom, Mikrobiom) wird aufgrund der rasant fortschreitenden biotechnologischen Entwicklung zukünftig möglich sein [8]. Unsere Gesundheitsdaten werden bald digital gespeichert und können bei Teilnahme an entsprechenden Exposomstudien direkt von den Prüfärzten in den Datensatz der Studie übertragen werden. Für die Handhabung und Analyse der riesigen Datenmengen („big data") werden Supercomputer verwendet, die selbstlernende bioinformatische Algorithmen anwenden, Interaktom- und Diseasome-Karten erstellen und über alle OMICs-Datensätze hinweg im Rahmen eines systembiologischen Gesamtansatzes auswerten, der Gesundheitsprognosen und präventive Maßnahmen für spezifische Umweltexpositionen erlauben wird. Einzig die ethischen und datenschutzrechtlichen Hürden müssen dann noch genommen werden, um dem Missbrauch des „gläsernen“ Probanden vorzubeugen.

\section{KURZGEFASST}

Um umweltbedingte Erkrankungen und Todesfälle besser zu untersuchen, wird eine „Environment-Wide Association Study“ (EWAS) analog zur genomweiten Assoziationsstudie (GWAS) gefordert.

\section{Das Exposom: die umfassende Beschreibung der lebenslangen Expositionshistorie}

Der Begriff „Exposom“ wurde erstmals im Jahr 2005 von Christopher P. Wild publiziert und im Detail beschrieben. Das Exposom repräsentiert die Summe aller lebenslangen Veränderungen der menschlichen Physiologie und Pathophysiologie durch Umweltfaktoren [10]. Wie bereits oben beschrieben umfassen diese Exposi- tionen nicht nur chemische und physikalische Faktoren wie Luftverschmutzung, Lärm, UV-Strahlung und klimatische Faktoren, sondern auch solche sozioökonomischer Natur wie das soziale und urbane Umfeld, aber auch die Exposition gegenüber Pathogenen, die alle der sog. „allgemeinen Umwelt/Umgebung“ zugeordnet werden. Der Lebensstil - sprich ob wir rauchen, übermäßig Alkohol trinken, uns gesund ernähren, körperlich aktiv sind und welche Konsumprodukte wir verwenden - spielt eine wesentliche Rolle für die sog. „spezifische Umwelt/Umgebung“ ( Das Exposom kann in beliebig viele „Pollutome“ unterteilt werden, die unter anderem nach dem Lebensabschnitt organisiert werden können (z. B. Stillzeit/Säugling, Kindheit, Jugend, Pubertät, Erwachsenenalter [inklusive Expositionen am Arbeitsplatz] und hohes Alter) [2]. Sprich die Pollutome repräsentieren zeitlich und örtlich einwirkende Expositionen und deren Auswirkungen auf die menschliche Physiologie bzw. Pathophysiologie.

Der Einfluss von Umweltstressoren ist besonders gravierend während der menschlichen Entwicklungsphase (z. B. im Fetus, bei Säuglingen, Kleinkindern und Kindern) und stellt ein weitgehend unbekanntes Feld dar, da Studien an diesen Individuen hohen ethischen Auflagen unterliegen. Allerdings könnten gerade diese Exposom-Studien von großem Interesse sein, da bereits geringste Expositionsgrade extreme Schäden auf die Entwicklung des Gehirns und anderer Organe verursachen können [12]. Expositionen während dieser Zeitfenster höchster Anfälligkeit im Frühstadium der Entwicklung könnten langfristige Gesundheitsschäden induzieren wie reduzierte kognitive Funktionen, aber auch psychische Veränderungen hin zur Gewalttätigkeit im Erwachsenenalter oder lebenslange ökonomische Unproduktivität (zusammengefasst in [2]). Die umweltbedingte fetale oder kindliche „Reprogrammierung“ bestimmter biologischer Vorgänge (z. B. über epigenetische Langzeitveränderungen) stellt dabei eine wesentliche sozioökonomische Herausforderung für unsere Gesellschaft dar. Auch der Beitrag von Umweltexpositionen auf das rasante Voranschreiten der Prävalenz neurodegenerativer Erkrankungen wie Alzheimer oder Parkinson ist bislang wenig untersucht, obwohl dieser Zusammenhang naheliegt [13]. Ausgewählte Beispiele für Exposom-Studien und -projekte sind in $>$ Abb. 2 aufgelistet.

\section{KURZGEFASST}

Das Exposom beschreibt die Änderungen der (patho)physiologischen Prozesse im Organismus und resultierende Krankheiten bzw. das Versterben durch die Summe aller Umweltexpositionen über die gesamte Lebensspanne.

\section{Auswirkungen von Umweltrisikofaktoren in der Frühphase des Lebens und im Alter}

Umweltrisikofaktoren tragen hauptsächlich in der Frühphase des Lebens und im hohen Alter zur Morbidität und Mortalität bei. Während die verlorenen gesunden Lebensjahre (DALYs) durch Umweltverschmutzung bei den Kleinkindern dominieren, ist die Anzahl der umweltbedingten Todesfälle in den Gruppen mit höherem Alter am höchsten ( $\triangleright$ Abb. 3) [2]. Die hohe Zahl der DALYs reflektiert vor allem die große Zahl verlorener Lebensjahre, wenn nur 


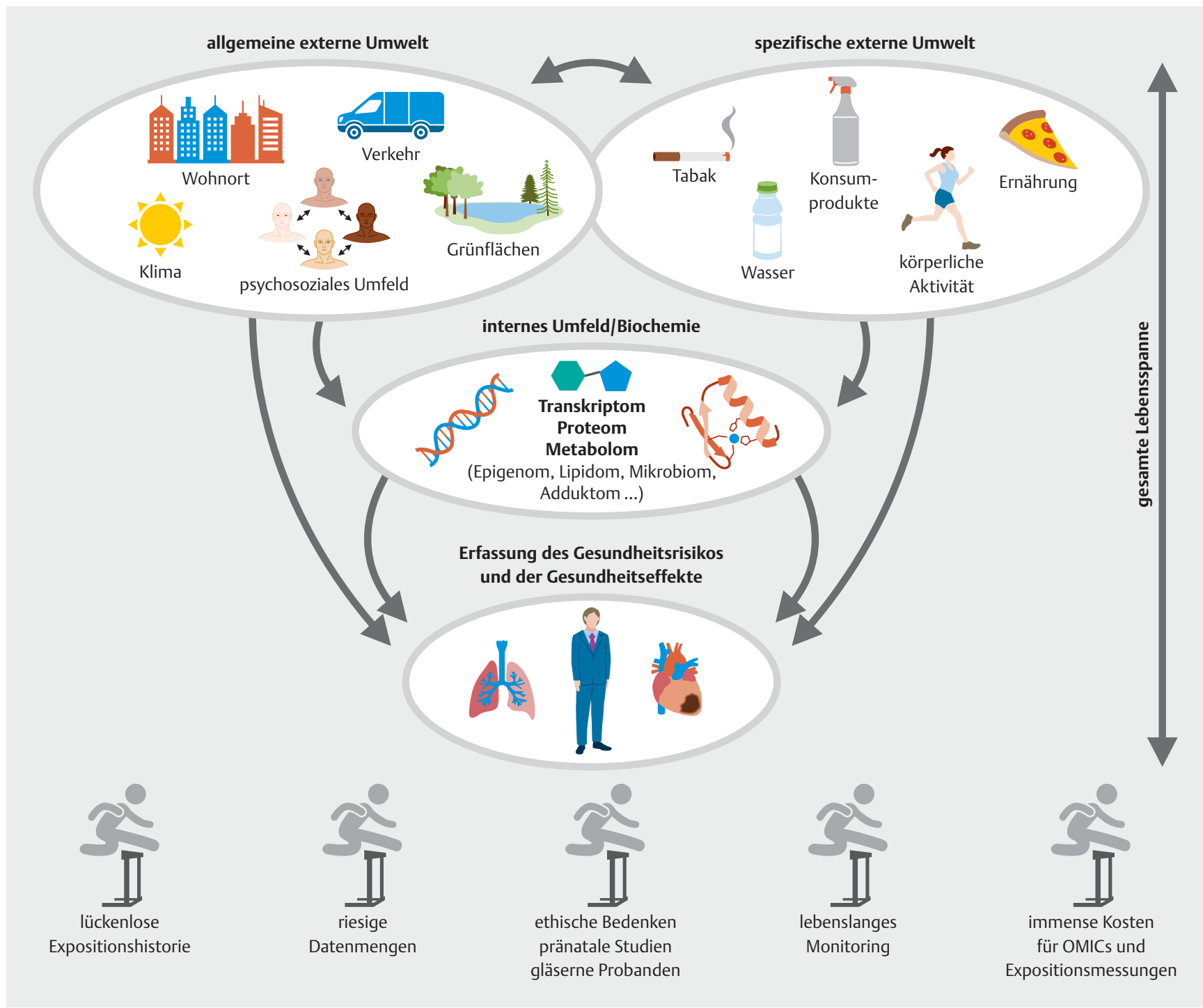

- Abb. 1 Das Exposom-Konzept. Das Exposom umfasst die Gesamtheit der lebenslangen Expositionen eines Individuums, von der Geburt (oder sogar der Zeit im Mutterleib) bis zum Tod. Dargestellt sind auch die größten Hürden für eine umfassende Exposom-Studie (EWAS). Quelle: Vrijheid M. The exposome: a new paradigm to study the impact of environment on health. Thorax 2014; 69: 876-878. doi:10.1136/thoraxjnl-2 013-204949. [rerif]

1 Kleinkind stirbt und die vielen Jahre, die Kinder mit schweren Krankheiten oder Einschränkungen leben müssen, wenn sie durch Umweltverschmutzung chronisch erkranken. Diese Daten sind im Einklang mit dem Exposom-Konzept, das auf das Studium der lebenslangen Expositionen und deren kontinuierliche Effekte auf unseren Organismus, Erkrankungen und Sterblichkeit abzielt. - Abb. 3 hebt auch hervor, dass Kleinkinder (< 4 Jahre) und ältere Menschen (> 60 Jahre) besonders vulnerable Gruppen darstellen, wenn es um umweltverschmutzungsbedingte DALYs und Sterblichkeit geht, die deshalb besonderen Schutz benötigen. Aus dem gleichen Grund stellen diese Bevölkerungsgruppen aber auch vorrangige Exposom-Studien-Populationen dar, da die dynamischen exposomischen Änderungen im Organismus in diesen extremen Altersgruppen offensichtlich besonders ausgeprägt sind.

Mögliche Erklärungen für die ausgeprägte umweltbedingte Übersterblichkeit im hohen Alter könnten sein, dass
1. Umweltexpositionen wie die chemische Verschmutzung Komorbiditäten für die altersbedingten klassischen Erkrankungen (Krebs, Herz-Kreislauf, Neurodegeneration) darstellen, die den natürlichen Alterungsprozess bzw. das Versterben beschleunigen;

2. die lebenslangen Expositionen an einem bestimmten Zeitpunkt im hohen Alter eskalieren, wenn der tolerable Schwellenwert der umweltbedingten akkumulierten biochemischen Schäden überschritten wird [8].

Mögliche Erklärungen für die ausgeprägten umweltbedingten DALYs im Kindesalter könnten sein, dass

1. Kinder weniger gut auf Umweltstressoren vorbereitet sind (geringere Resilienz), da der Organismus während der Lebensspanne durch kontinuierliche niedriggradige Expositionen Mechanismen entwickelt, um die daraus resultierenden Schäden zu 


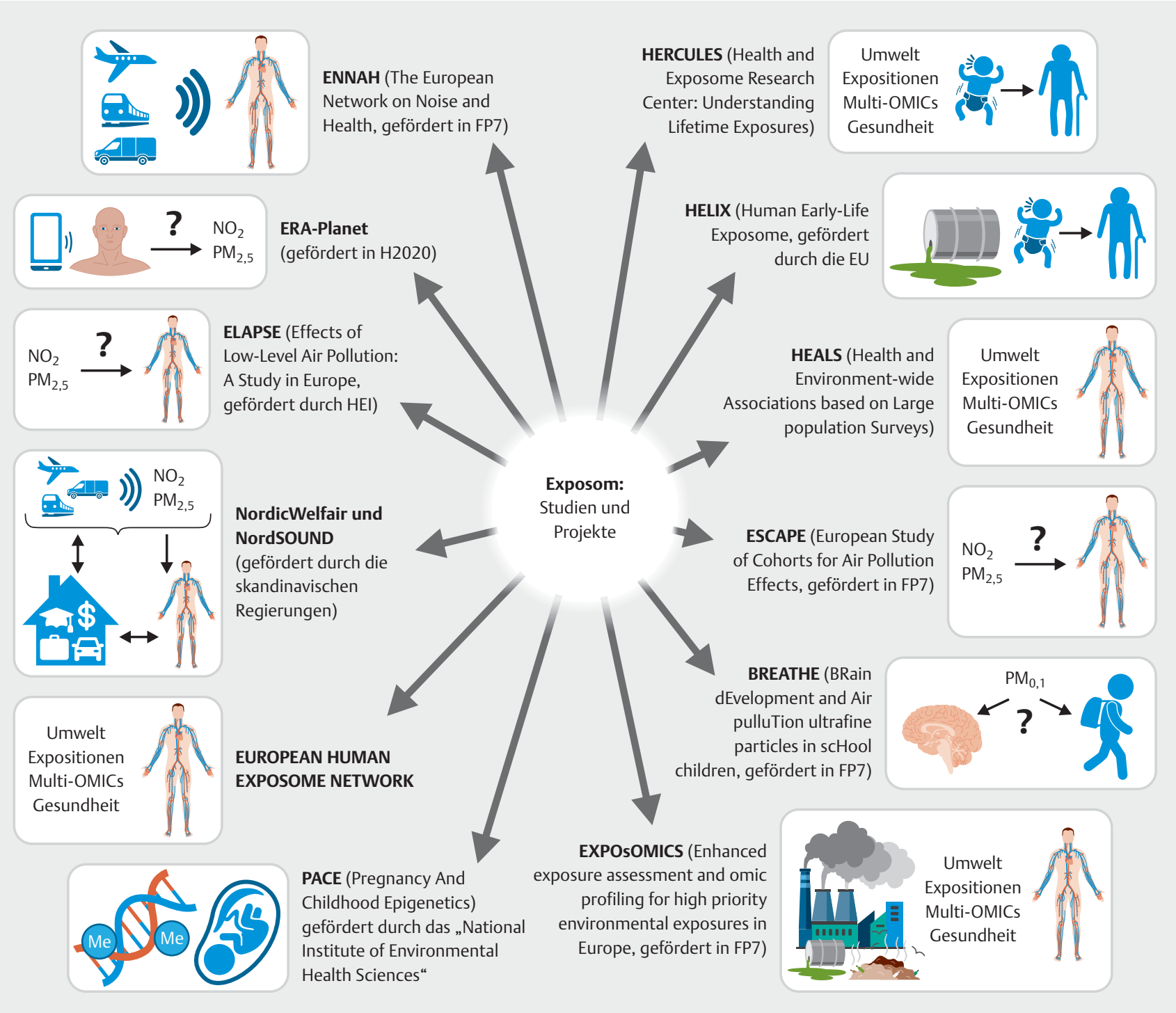

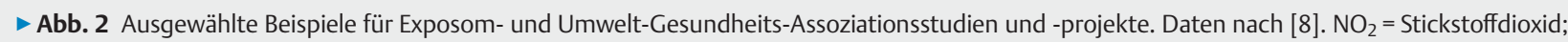
PM2,5 = Feinstaub mit Durchmesser $\leq 2,5 \mu \mathrm{m}$; PM0,1 = Ultrafeinstaub mit Durchmesser $\leq 0,1 \mu \mathrm{m} ; \mathrm{FP7}=7$. Forschungsrahmenprogramm der Europäischen Kommission; H2020 = EU-Forschungsrahmenprogramm Horizon 2020; HEI = Health Effects Institute

kompensieren bzw. zu reparieren (klassisches Stress-AntwortPrinzip wie bei jeder Art der Präkonditionierung);

2. Kinder höheren Dosen aufgrund des höheren KonzentrationsKörpergewichts-Verhältnisses ausgesetzt werden;

3. eine unvorteilhafte fetale Reprogrammierung (z. B. durch ungesunde Lebensweise oder Lebensraum der Schwangeren) besteht;

4. pränatale und frühkindliche Entwicklungsprozesse Zellen und Organe anfälliger machen für Umweltgifte und -stressoren [8].

\section{KURZGEFASST}

Kleinkinder und alte Menschen sind besonders vulnerabel gegenüber den schädlichen Folgen durch Umweltexpositionen.

\section{Ausblick und zukünftige Strategien}

Große Exposom-Studien liefern erste wertvolle Daten zum Beitrag von Umweltrisikofaktoren zur Gesundheitsbelastung der Bevölkerung. Aber detaillierte Daten zur Wirkung einzelner Umweltstressoren auf spezifische Krankheiten oder Mortalität werden erst verfügbar sein, wenn die Summe der Umweltexpositionen sehr genau 
verlorene gesunde Lebensjahre (DALYs)

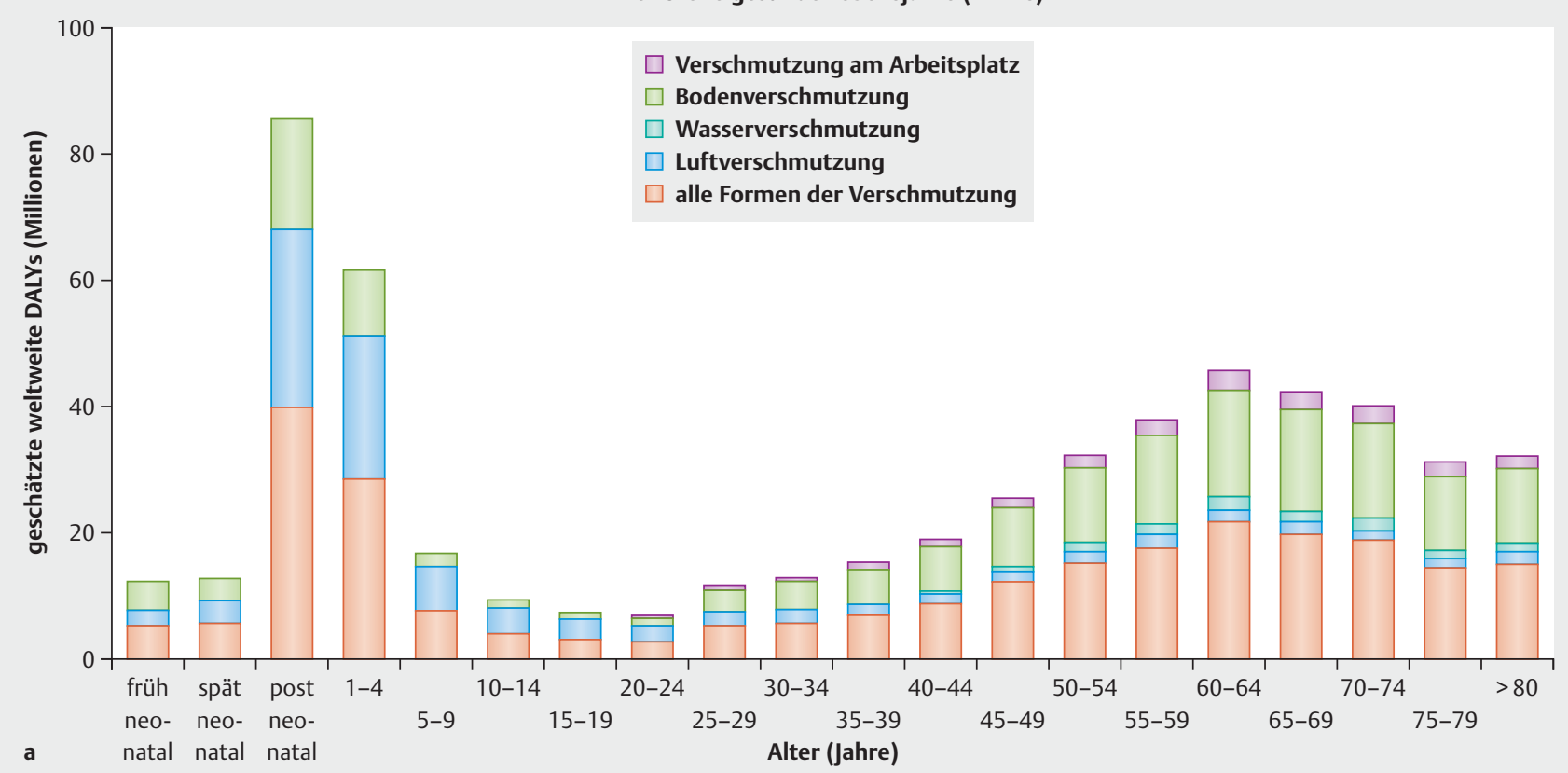

Todesfälle

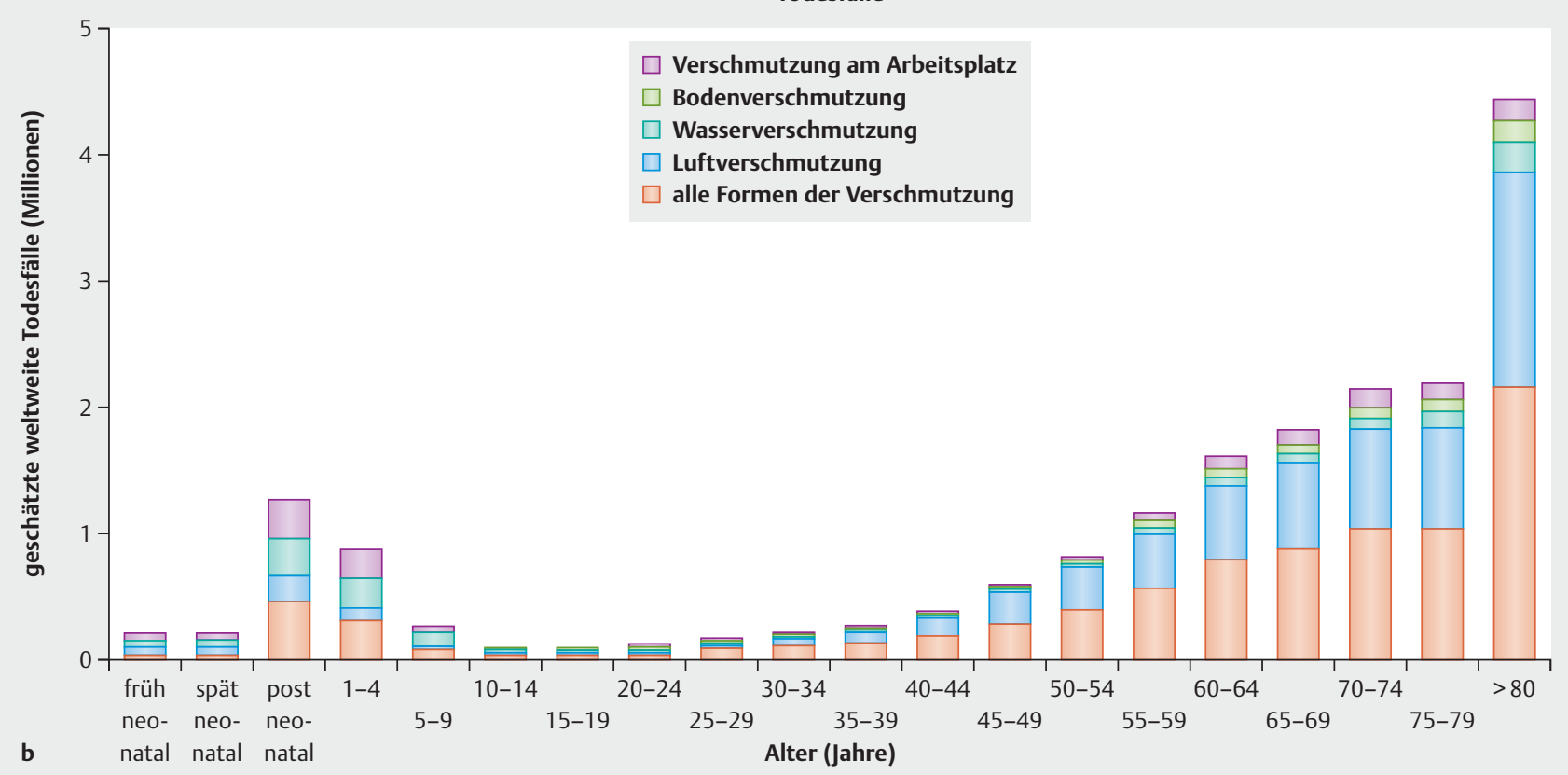

- Abb. 3 Geschätzte weltweite DALYs (a) und Todesfälle (b) durch verschiedene Formen der Umweltverschmutzung in Abhängigkeit vom Alter. Diagramme gezeichnet mit Daten aus der Global Burden of Disease-Studie und dem Bericht der Lancet Commission on Pollution and Health basierend auf dem Stand im Jahr 2015. Daten nach [2, 14].

erfasst werden kann, d.h. unter anderem die zeitlich aufgelöste Bestimmung der Konzentrationen von Umweltgiften in Luft/Wasser/Boden, die kontinuierliche Messung von Schallpegel/Temperatur/UV-Strahlung sowie wiederholende Abfragen mentaler/sozialer Faktoren wie Stress am Arbeitsplatz oder Vereinsamung mithilfe von Fragebögen bzw. Smartphone-Anwendungen. Daneben müssten in regelmäßigen Abständen (idealerweise monatlich oder besser wöchentlich) unsere biologischen und biochemischen Parameter bestimmt werden (d.h. aufwendige Metabolom-, Proteomund Transkriptom-[OMICs-]Analysen), um die Veränderungen die- 
ser Systeme permanent zu erfassen und mit den erfassten Expositionen zu korrelieren. Um ein ganzheitliches Bild zu erhalten, wie Umweltrisikofaktoren unseren Organismus und unsere Gesundheit beeinflussen, müssten diese Messungen der Expositionen und Änderungen unserer körpereigenen Systeme über die gesamte Lebensspanne erfolgen, idealerweise bereits im Mutterleib beginnend und mit dem Tod endend. Es ist sehr gut verständlich, dass bereits für die Erfassung all dieser Parameter über die gesamte Lebensspanne eines einzelnen Individuums nicht nur unvorstellbare Datenmengen anfallen würden, sondern zum derzeitigen Stand auch astronomische Kosten entstehen würden, die in Kohorten von mehreren tausend Teilnehmern sich kein Land auf der Welt leisten könnte. Daneben wird es hohe ethische Ansprüche an derartige Studien hinsichtlich des Datenschutzes der „gläsernen“ Probanden geben.

\section{Fazit}

Es ist zu erwarten, dass die genannten Hürden betreffs der Exposom-Forschung in Zukunft überwunden werden, da der technologische Fortschritt zum einen die Handhabung riesiger Datenmengen ermöglichen wird und andererseits die Kosten für derart umfangreiche OMICs-Analysen dramatisch verringern wird. Sobald ethische Bedenken ausgeräumt sind, könnte eine allumfassende weltweite Exposom-Studie (EWAS) den Beitrag einzelner Umweltrisikofaktoren zur Krankheitsbelastung und Mortalität revolutionieren und durch gezielte präventive Maßnahmen unvorstellbare Einsparungen im globalen Gesundheitssystem bewirken.

Fördermittel

"Novel and neglected cardiovascular risk factors: | Boehringer Ingelheim Stiftung | https://www.boehringer-ingelheim-stiftung.de/waswir-foerdern/initiativen-fuer-die-region/initiativen-an-der-unimedizinmainz.html

Mainzer Herz 17 und 21 | Stiftung Mainzer Herz | http://www.herzstiftung-mainzer-herz.de/herzstiftung/startseite/uebersicht.html

Partner Rhein-Main, Standort Mainz | Deutsches Zentrum für HerzKreislaufforschung | https://dzhk.de/

Interessenkonflikt

Die Autorinnen/Autoren geben an, dass kein Interessenkonflikt besteht.
Literatur

[1] Lozano R, Naghavi M, Foreman K et al. Global and regional mortality from 235 causes of death for 20 age groups in 1990 and 2010: a systematic analysis for the Global Burden of Disease Study 2010. Lancet 2012; 380: 2095-2128. doi:10.1016/50140-6736(12)61728-0

[2] Landrigan PJ, Fuller R, Acosta NJR et al. The Lancet Commission on pollution and health. Lancet 2018; 391: 462-512. doi:10.1016/S0140-6736(1 7) $32345-0$

[3] GBD 2015 DALYs and HALE Collaborators. Global, regional, and national disability-adjusted life-years (DALYs) for 315 diseases and injuries and healthy life expectancy (HALE), 1990-2015: a systematic analysis for the Global Burden of Disease Study 2015. Lancet 2016; 388: 1603-1658. doi:10.1016/S0140-6736(16)31460-X

[4] Munzel T, Daiber A. Environmental Stressors and Their Impact on Health and Disease with Focus on Oxidative Stress. Antioxid Redox Signal 2018; 28: 735-740. doi:10.1089/ars.2017.7488

[5] Li H, Kilgallen AB, Munzel T et al. Influence of mental stress and environmental toxins on circadian clocks: Implications for redox regulation of the heart and cardioprotection. Br J Pharmacol 2020; 177: 5393-5412. doi:10.1111/bph.14949

[6] Rappaport SM. Genetic Factors Are Not the Major Causes of Chronic Diseases. PLoS One 2016; 11: e0154387. doi:10.1371/journal.pone.01543 87

[7] Sainani K. Taking on the Exposome - Bringing Bioinformatics Tools to the Environmental Side of the Health Equation. Biomed Comput Rev 2016; 2016: 14-21

[8] Daiber A, Lelieveld J, Steven S et al. The "exposome" concept - how environmental risk factors influence cardiovascular health. Acta Biochim Pol 2019; 66: 269-283. doi:10.18388/abp.2019_2853

[9] Munzel T, Sorensen M, Gori T et al. Environmental stressors and cardiometabolic disease: part II-mechanistic insights. Eur Heart J 2017; 38: 557-564. doi:10.1093/eurheartj/ehw294

[10] Wild CP. Complementing the genome with an "exposome": the outstanding challenge of environmental exposure measurement in molecular epidemiology. Cancer Epidemiol Biomarkers Prev 2005; 14: 18471850. doi:10.1158/1055-9965.EPI-05-0456

[11] Vrijheid M. The exposome: a new paradigm to study the impact of environment on health. Thorax 2014; 69: 876-878. doi:10.1136/thoraxjnl-2 013-204949

[12] Bellinger DC. Prenatal Exposures to Environmental Chemicals and Children's Neurodevelopment: An Update. Saf Health Work 2013; 4: 1-11. doi:10.5491/SHAW.2013.4.1.1

[13] Chen $\mathrm{H}$, Kwong JC, Copes $\mathrm{R}$ et al. Living near major roads and the incidence of dementia, Parkinson's disease, and multiple sclerosis: a population-based cohort study. Lancet 2017; 389: 718-726. doi:10.1016/S014 0-6736(16)32399-6

[14] GBD 2015 Risk Factors Collaborators. Global, regional, and national comparative risk assessment of 79 behavioural, environmental and occupational, and metabolic risks or clusters of risks, 1990-2015: a systematic analysis for the Global Burden of Disease Study 2015. Lancet 2016; 388: 1659-1724. doi:10.1016/S0140-6736(16)31679-8 